\title{
DOMINATION, UNIQUENESS AND REPRESENTATION THEOREMS FOR HARMONIC FUNCTIONS IN HALF-SPACES
}

\author{
D. H. ARMITAGE
}

We shall use results of the author [1] on half-space Poisson integrals and a recent measure-theoretic result of Watson [11]* to deduce theorems of the types described in the title.

Let $D$ denote the Euclidean half-space $\boldsymbol{R}^{n} \times(0,+\infty)$, where $n \geqq 1$, and let $\partial D$ denote the Euclidean boundary of $D$. Arbitrary points of $D$ and $\partial D$ are denoted by $M=(X, x)$ and $P=(T, 0)$, respectively, where $X, T \in \boldsymbol{R}^{n}$ and $x \in(0,+\infty)$. We write $|X|$ and $|M|$ for the Euclidean norms of $X$ and $M$. The origins of $\boldsymbol{R}^{n}$ and $\boldsymbol{R}^{n+1}$ are denoted by $\mathcal{O}^{*}$ and $\mathcal{O}$, respectively.

If $\mu$ is a signed measure on $\partial D$ such that

$$
\int_{\partial D}(1+|P|)^{-n-1} d|\mu|(P)<+\infty
$$

then the Poisson integral $I_{\mu}$ of $\mu$ is defined in $D$ by the equation

$$
I_{\mu}(M)=2\left(s_{n+1}\right)^{-1} \int_{\partial D} x|M-P|^{-n-1} d \mu(P)
$$

where $s_{n+1}$ is the surface area of the unit sphere in $\boldsymbol{R}^{n+1}$. The condition (1) is necessary and sufficient for $I_{\mu}$ to be harmonic in $D$ (see Flett [6; Theorem 6]), and we shall say that $\mu$ is of class $\mathscr{F}$ if (1) is satisfied. If, further, $\mu$ is non-negative, we write $\mu \in \mathscr{F}^{+}$.

We shall be concerned with a class of harmonic functions in $D$ which can be characterized in various ways; recall the following:

Lemma A. Let $u$ be harmonic in D. The following are equivalent:

(i) $u$ is the difference of two non-negative harmonic functions in $D$,

(ii) there exists a unique measure $\mu \in \mathscr{F}$ and a unique real number $c$ such that

$$
u(M)=I_{\mu}(M)+c x \quad(M \in D)
$$

* I am grateful to Dr. Watson for a pre-print of his paper. 
(iii) $u^{+}$has a harmonic majorant in $D$,

(iv) the function

$$
x \rightarrow \int_{R^{n}}\left(|X|^{2}+(x+1)^{2}\right)^{-(n+1) / 2} u^{+}(X, x) d X
$$

is bounded on $(0,+\infty)$.

If $u \geqq 0$ in $D$, the measure $\mu$ and the constant c occurring in (2) are non-negative.

The assertion in the last paragraph of the lemma and the equivalence of (i) and (ii) are easy consequences of the Poisson integral representation theorem for non-negative harmonic functions in $D$, due to Tsuji for $n=1$ (see, for example, [10, p. 149]) and to Dinghas [5] in the general case (see also Kuran [7]). The equivalence of (i) and (iii) is obvious, and the equivalence of (iii) and (iv) follows, since $u^{+}$is subharmonic in $D$, from a result of Kuran [8].

If $u$ is harmonic in $D$ and satisfies the equivalent conditions in Lemma A, we shall say that $u$ is of class $\mathscr{H}$. If $u$ is non-negative and harmonic in $D$, we write $u \in \mathscr{H}^{+}$.

For each point $P$ of $\partial D$ and each positive number $r$, we write

$$
\sigma(P, r)=\{M \in D:|M-P|=r\}
$$

and

$$
\tau(P, r)=\{Q \in \partial D:|Q-P|<r\}
$$

and we denote surface-area measure on $\sigma(P, r)$ by $s$.

Recall that if $u \in \mathscr{H}$, then for each $P \in \partial D$ the half-spherical "mean", given by

$$
\mathscr{M}(u, P, r)=r^{-n-2} \int_{\sigma(P, r)} x u(M) d s(M) \quad(r>0),
$$

is real-valued and continuous on $(0,+\infty)$. (See [2] for references and for further properties of the mean.) In [1, Lemma 1, Theorem 1] we proved the following results which are essential for the proofs in the present paper.

Lemma B. If $v \in \mathscr{F}+$ and $P=(T, 0) \in \partial D$, then the following are equivalent:

(i) $\int_{0}^{1} t^{-n-2} v(\tau(P, t)) d t=+\infty$,

(ii) $\lim _{y \rightarrow 0+} y^{-1} I_{v}(T, y)=+\infty$,

(iii) $\lim _{r \rightarrow 0+} \mathscr{M}\left(I_{v}, P, r\right)=+\infty$. 
Theorem A. Suppose that $\mu \in \mathscr{F}$ and $v \in \mathscr{F}+$. If $P=(T, 0) \in \partial D$ and one of the conditions (i), (ii) and (iii) in Lemma $B$ is satisfied, then

$$
\begin{aligned}
& \liminf _{r \rightarrow 0+} \frac{\mu(\tau(P, r))}{v(\tau(P, r))} \leqq \liminf _{r \rightarrow 0+} \frac{\mathscr{M}\left(I_{\mu}, P, r\right)}{\mathscr{M}\left(I_{v}, P, r\right)} \\
& \leqq \liminf _{y \rightarrow 0+} \frac{I_{\mu}(T, y)}{I_{v}(T, y)} \leqq \limsup _{y \rightarrow 0+} \frac{I_{\mu}(T, y)}{I_{v}(T, y)} \\
& \leqq \limsup _{r \rightarrow 0+} \frac{\mathscr{M}\left(I_{\mu}, P, r\right)}{\mathscr{M}\left(I_{v}, P, r\right)} \leqq \limsup _{r \rightarrow 0+} \frac{\mu(\tau(P, r))}{v(\tau(P, r))} .
\end{aligned}
$$

The measure-theoretic result we require was proved by Watson [11, Theorem 1] and depends on a theorem of Besicovitch [3, Theorem 3].

Theorem B. Let $\mu$ and $v$ be measures on $\partial D$ such that $v(\tau(P, r))>0$ for each $P$ in $\partial D$ and each positive $r$. If

$$
\limsup _{r \rightarrow 0+} \frac{\mu(\tau(P, r))}{v(\tau(P, r))}
$$

is greater than $-\infty$ for all $P$ in $\partial D$ and is non-negative for $v$-almost all $P$ in $\partial D$, then $\mu$ is non-negative.

1. An extension of Theorem A. In order to make Theorem A applicable to functions of class $\mathscr{H}$, we require the following result which is analogous to Theorem A but which concerns behaviour "near infinity".

Lemma 1. Suppose that $u, v \in \mathscr{H}$ and that

$$
u(M)=I_{\mu}(M)+c x \quad(M \in D)
$$

and

$$
v(M)=I_{v}(M)+d x \quad(M \in D),
$$

where $\mu, v \in \mathscr{F}$ and $c, d$ are real constants. If $c$ and $d$ are not both zero, then

$$
\lim _{y \rightarrow \infty} \frac{u\left(\mathcal{O}^{*}, y\right)}{v\left(\mathcal{O}^{*}, y\right)}=\lim _{r \rightarrow \infty} \frac{\mathscr{M}(u, \mathcal{O}, r)}{\mathscr{M}(v, \mathcal{O}, r)}=\frac{c}{d},
$$

where $c / d$ is understood to be $+\infty$ (resp. - $-\infty)$ if $d=0<c \quad($ resp. $>c)$.

By [1, Lemma 4],

$$
I_{\mu}\left(\mathcal{O}^{*}, y\right)=(2 n+2)\left(s_{n+1}\right)^{-1} y \int_{0}^{\infty} t\left(y^{2}+t^{2}\right)^{-(n+3) / 2} \mu(\tau(\mathcal{O}, t)) d t .
$$

Also, by [1, Lemma 2],

$$
\int_{0}^{\infty}(1+t)^{-n-2}|\mu|(\tau(\mathcal{O}, t)) d t<+\infty
$$


Hence, if $\varepsilon>0$, then there exists a positive number $A$ such that when $y>1$

$$
\begin{aligned}
& \quad\left|\int_{0}^{\infty} t\left(y^{2}+t^{2}\right)^{-(n+3) / 2} \mu(\tau(\mathcal{O}, t)) d t\right| \\
& \leqq \int_{0}^{A} t\left(y^{2}+t^{2}\right)^{-(n+3) / 2}|\mu|(\tau(\mathcal{O}, t)) d t+\int_{A}^{\infty} t\left(1+t^{2}\right)^{-(n+3) / 2}|\mu|(\tau(\mathcal{O}, t)) d t \\
& <\frac{1}{2} A^{2}|\mu|(\tau(\mathcal{O}, A)) y^{-n-3}+\varepsilon .
\end{aligned}
$$

It follows that as $y \rightarrow+\infty$

$$
u\left(\mathcal{O}^{*}, y\right)=I_{\mu}\left(\mathcal{O}^{*}, y\right)+c y=(c+o(1)) y .
$$

Using this and the corresponding result for $v$, we find that the first limit in (5) exists when $c$ and $d$ are not both zero and has the stated value.

Since $\mathscr{M}\left(I_{\mu}, \mathcal{O}, r\right) \rightarrow 0$ as $r \rightarrow+\infty$ by [1, Lemma 2(iii)] and similarly for $I_{v}$, the corresponding result for the second limit in (5) follows easily from the observation that $\mathscr{U}(x, \mathcal{O}, \cdot)$ is a positive constant on $(0,+\infty)$.

\section{Domination and uniqueness theorems}

Theorem 1. Suppose that $u \in \mathscr{H}, v \in \mathscr{H}^{+}$and

$$
\lim _{r \rightarrow 0+} \mathscr{M}(v, P, r)=+\infty
$$

for each $P$ in $\partial D$. If

$$
\limsup _{r \rightarrow 0+} \frac{\mathscr{M}(u, P, r)}{\mathscr{M}(v, P, r)}>-\infty
$$

for each $P$ in $\partial D$ and if there exists a real number $A$ such that

$$
\limsup _{r \rightarrow 0+} \frac{\mathscr{M}(u, P, r)}{\mathscr{M}(v, P, r)} \geqq A
$$

for v-almost all $P$ in $\partial D$, where $v$ is the measure occurring in the Poisson integral representation of $v$, and if

then $u \geqq A v$ in $D$.

$$
\limsup _{r \rightarrow \infty} \frac{\mathscr{M}(u, \mathcal{O}, r)}{\mathscr{M}(v, \mathcal{O}, r)} \geqq A
$$

In proving this theorem, we may suppose that $A=0$, for otherwise we could work with $u-A v$. Suppose that $u$ and $v$ have the representations (3) and (4). Since $\mathscr{M}(x, P, \cdot)$ is constant on $(0,+\infty)$, equation (6) implies that

$$
\lim _{r \rightarrow 0+} \mathscr{M}\left(I_{v}, P, r\right)=+\infty \quad(P \in \partial D)
$$


and that

$$
\limsup _{r \rightarrow 0+} \frac{\mathscr{M}(u, P, r)}{\mathscr{M}(v, P, r)}=\limsup _{r \rightarrow 0+} \frac{\mathscr{M}\left(I_{\mu}, P, r\right)}{\mathscr{M}\left(I_{v}, P, r\right)} \quad(P \in \partial D) .
$$

Hence, by Theorem A,

$$
\limsup _{r \rightarrow 0+} \frac{\mu(\tau(P, r))}{v(\tau(P, r))}
$$

is greater than $-\infty$ for all $P$ in $\partial D$ and is non-negative for $v$-almost all $P$ in $\partial D$. By (7) and Lemma B

$$
\int_{0}^{1} t^{-n-2} v(\tau(P, t)) d t=+\infty \quad(P \in \partial D)
$$

and therefore $v(\tau(P, r))>0$ for each $P$ in $\partial D$ and each positive $r$. Hence Theorem $\mathrm{B}$ is applicable, and we find that $\mu$ is non-negative. Hence $I_{\mu} \geqq 0$ in $D$, and it remains to prove that $c \geqq 0$. If $c=0$, there is nothing to prove, and if $c \neq 0$, then, since $d \geqq 0$, the required result follows from Lemma 1 .

In Theorem 1 all the hypotheses on the behaviour of mean values can be replaced by hypotheses concerning behaviour along normals to $\partial D$. Thus, modifying the proof of Theorem 1 in obvious ways, we obtain the following.

Theorem 2. Suppose that $u \in \mathscr{H}, v \in \mathscr{H}^{+}$and

for each $T$ in $\boldsymbol{R}^{n}$. If

$$
\lim _{y \rightarrow 0+} y^{-1} v(T, y)=+\infty
$$

$$
\limsup _{y \rightarrow 0+} \frac{u(T, y)}{v(T, y)}>-\infty
$$

for each $T$ in $\boldsymbol{R}^{n}$ and if there exists a real number $A$ such that

$$
\limsup _{y \rightarrow 0+} \frac{u(T, y)}{v(T, y)} \geqq A
$$

for $v$-almost all $(T, 0)$ in $\partial D$, where $v$ is the measure occurring in the Poisson integral representation of $v$, and if

then $u \geqq A v$ in $D$.

$$
\limsup _{y \rightarrow \infty} \frac{u\left(\mathcal{O}^{*}, y\right)}{v\left(\mathcal{O}^{*}, y\right)} \geqq A,
$$

In fact, all our results with hypotheses on mean values have counterparts with hypotheses on behaviour along normals. Because of the penultimate inequality in Theorem A, the mean value hypotheses seem better, and henceforth we give our results only in their "mean value" form and take for granted the corresponding" "normal" form. 
We next consider a special case of Theorem 1 . In order to state it in its simplest form, we need the following lemma. The $n$-dimensional Lebesgue measure on $\partial D$ is denoted by $\lambda$.

Lemma 2. If $u \in \mathscr{H}$, then

$$
\lim _{r \rightarrow \infty} \mathscr{M}(u, \mathcal{O}, r)
$$

exists and is finite and

$$
\lim _{r \rightarrow 0+} r \mathscr{M}(u, P, r)
$$

exists and is finite for $\lambda$-almost all $P$ in $\partial D$.

The first result in the lemma follows by taking $v(M)=x(M \in D)$ in Lemma 1 .

The second result follows by taking $v \equiv 1$ in Theorem $\mathrm{A}$ and observing that $r^{-n} \mu(\tau(P, r))$ has a finite limit as $r \rightarrow 0+$ for $\lambda$-almost all $P$ in $\partial D$.

Theorem 3. Suppose that $u \in \mathscr{H}$. If

$$
\lim _{r \rightarrow 0+} r \mathscr{M}(u, P, r)
$$

is non-negative for $\lambda$-almost all $P$ in $\partial D$ and is not equal to $-\infty$ for any $P$ in $\partial D$ and if

$$
\lim _{r \rightarrow \infty} \mathscr{M}(u, \mathcal{O}, r) \geqq 0,
$$

then $u \geqq 0$ in $D$.

This is obtained by taking $v(M)=1+x(M \in D)$ and $A=0$ in Theorem 1. The corresponding result for the unit disc (with hypotheses on the radial limits) is due to Bruckner, Lohwater and Ryan [4; Theorem 2].

We come now to the uniqueness results. These are applications of Theorem 1.

The orem 4. Suppose that $u \in \mathscr{H}$ and $v \in \mathscr{H}^{+}$. Suppose also that (6) holds for each $P$ in $\partial D$. If

$$
\liminf _{r \rightarrow 0+} \frac{|\mathscr{M}(u, P, r)|}{\mathscr{M}(v, P, r)}<+\infty
$$

for each $P$ in $\partial D$,

$$
\liminf _{r \rightarrow 0+} \frac{\mathscr{M}(u, P, r)}{\mathscr{M}(v, P, r)}=0
$$

for v-almost all $P$ in $\partial D$, where $v$ is the measure occurring in the Poisson integral representation of $v$, and

$$
\liminf _{r \rightarrow \infty} \frac{\mathscr{M}(u, \mathcal{O}, r)}{\mathscr{M}(v, \mathcal{O}, r)}=0
$$

then $u \equiv 0$ in $D$.

By applying Theorem 1 with $-u$ replacing $u$ and with $A=0$, we obtain $u \leqq 0$ in $D$. Hence we can replace the lower limits in (8) and (9) by limits. Another application of Theorem 1 now shows that $u \geqq 0$ in $D$, and the result is proved. 
If we take $v(M)=1+x(M \in D)$ in Theorem 4, then, in view of Lemma 2 we obtain the following.

Theorem 5. Suppose that $u \in \mathscr{H}$. If $\lim _{r \rightarrow 0+} r \mathscr{M}(u, P, r)$ is zero for $\lambda$-almost all $P$ in $\partial D$ and is not equal to $\pm \infty$ for any $P$ in $\partial D$ and if $\mathscr{M}(u, \mathcal{O}, r) \rightarrow 0$ as $r \rightarrow \infty$, then $u \equiv 0$ in $D$.

Alternatively, Theorem 5 can be proved by applying Theorem 3 to $u$ and $-u$.

The corresponding result for the unit disc (with hypotheses on the radial limits) is due to Lohwater [9; Corollary].

In fact, we can weaken the hypotheses in Theorem 5. For this, we need the following lemmas.

Lemma 3. Let $E$ be a set of $\lambda$-measure 0 in $\partial D$. Then there exists a positive harmonic function $v$ in $D$ such that

for each $P$ in $E$.

$$
\lim _{r \rightarrow 0+} r \mathscr{M}(v, P, r)=\lim _{\substack{M \rightarrow P \\ M \in D}} v(M)=+\infty
$$

Lemma 4. For each $P$ in $\partial D$ and each positive number $r$

$$
\mathscr{M}(1, P, r)=\varkappa_{n} r^{-1}
$$

and

$$
\mathscr{M}(x, P, r)=(2 n+2)^{-1} s_{n+1},
$$

where $\chi_{n}$ is the volume of the unit ball in $\boldsymbol{R}^{n}$.

The results in Lemma 4 can be proved by direct calculation. For an aid to the calculation entailed in proving (11), see [7; p. 285].

In proving Lemma 3 , we note first that it is enough to produce a positive harmonic function $v$ in $D$ which satisfies the second equation in (10) for each $P$ in $E$, for we always have, by Lemma 4,

$$
\begin{aligned}
\liminf _{r \rightarrow 0+} r \mathscr{M}(v, P, r) & \geqq r \mathscr{M}(1, P, r) \liminf _{\substack{M \rightarrow P \\
M \in D}} v(M) \\
& =x_{n} \liminf _{\substack{M \rightarrow P \\
M \in D}} v(M) .
\end{aligned}
$$

Let $\left(\Omega_{m}\right)$ be a sequence of relatively open subsets of $\partial D$ such that $\Omega_{m} \supset E$ and $\lambda\left(\Omega_{m}\right)<2^{-m}$ for each $m$. For each $m$, let $v_{m}$ be the Poisson integral in $D$ of the characteristic function of $\Omega_{m}$, and let $v=\sum_{m=1}^{\infty} v_{m}$ in $D$. By well known properties of Poisson integrals, each $v_{m}$ is harmonic and positive in $D$ and $v_{m}(M) \rightarrow 1$ as $M \rightarrow P$ for each $P$ in $\Omega_{m}$. It follows that $v$ satisfies the second equation in (10) for each $P$ 
in $E$ and that $v$ is either positive and harmonic in $D$ or identically equal to $+\infty$ in $D$. The latter alternative is excluded since

$$
\frac{1}{2} s_{n+1} \sum_{m=1}^{\infty} v_{m}\left(\mathcal{O}^{*}, 1\right)<\sum_{m=1}^{\infty} \lambda\left(\Omega_{m}\right)<\sum_{m=1}^{\infty} 2^{-m}=1 .
$$

Hence $v$ has the required properties.

Our improvement of Theorem 5 will be obtained as a corollary of the following result.

Theorem 6. Suppose that $u \in \mathscr{H}$ and that there exist real numbers $A$ and $B$ such that

$$
\liminf _{r \rightarrow 0+} r \mathscr{M}(u, P, r) \leqq A \varkappa_{n}
$$

for all $P$ in $\partial D \backslash E$, where $\lambda(E)=0$, and

$$
\liminf _{r \rightarrow \infty} \mathscr{M}(u, \mathcal{O}, r) \leqq B(2 n+2)^{-1} s_{n+1} .
$$

Let $v$ be a positive harmonic function in D. If

$$
\liminf _{r \rightarrow 0+} \frac{\mathscr{M}(u, P, r)}{\mathscr{M}(v, P, r)} \leqq 0
$$

for each $P$ in $E$, then $u(M) \leqq A+B x$ for each $M$ in $D$.

Notice first that (12) will continue to hold if $v$ is increased by the addition of another function which is positive and harmonic in $D$. Hence, by Lemma 3, we may suppose that $r \mathscr{M}(v, P, r) \rightarrow+\infty$ as $r \rightarrow 0+$ for each $P$ in $E$. Further, by adding the function $1+x$ to $v$, we may suppose also that $\mathscr{M}(v, P, r) \rightarrow+\infty$ as $r \rightarrow 0+$ for each $P$ in $\partial D$ and that

$$
\liminf _{r \rightarrow \infty} \mathscr{M}(v, \mathcal{O}, r)>0
$$

(see Lemma 4). Also, we may suppose that $A=B=0$, since, by Lemma 4, we could replace $u$ throughout by the function $M \sim u(M)-A-B x$. It follows from the hypotheses and the properties of $v$ that

for each $P$ in $\partial D$ and that

$$
\liminf _{r \rightarrow 0+} \frac{\mathscr{M}(u, P, r)}{\mathscr{M}(v, P, r)} \leqq 0
$$

$$
\liminf _{r \rightarrow \infty} \frac{\mathscr{M}(u, \mathcal{O}, r)}{\mathscr{M}(v, \mathcal{O}, r)} \leqq 0 .
$$

The result now follows from Theorem 1.

Corollary. Suppose that $u \in \mathscr{H}$. Suppose also that $r \mathscr{M}(u, P, r) \rightarrow 0$ as $r \rightarrow 0+$ for each $P$ in $\partial D \backslash E$, where $\lambda(E)=0$, and that $\mathscr{M}(u, \mathcal{O}, r) \rightarrow 0$ as $r \rightarrow \infty$. If there exists a positive harmonic function $v$ in $D$ such that $\mathscr{M}(u, P, r) / \mathscr{M}(v, P, r) \rightarrow 0$ as $r \rightarrow 0+$ for each $P$ in $E$, then $u \equiv 0$ in $D$. 
The Corollary follows by applying Theorem 6 to $u$ and $-u$. It follows from Lemma 3 that this Corollary is stronger than Theorem 5.

3. A representation theorem. Here we use Theorem 6 to obtain a representation theorem for functions of class $\mathscr{H}$. An analogous result for the unit disc was proved by Bruckner, Lohwater and Ryan [4, Theorem 3].

Theorem 7. Suppose that $u \in \mathscr{H}$. If

$$
\lim _{r \rightarrow 0+} r \mathscr{M}(u, P, r)
$$

is non-negative for $\lambda$-almost all $P$ in $\partial D$ and is equal to $-\infty$ only for a countable set $\left\{P_{1}, P_{2}, \ldots\right\}$ of points in $\partial D$ and if

$$
\limsup _{r \rightarrow \infty} \mathscr{M}(u, \mathcal{O}, r)=k,
$$

then

$$
u(M)=I_{\mu_{1}}(M)-I_{\mu_{2}}(M)+(2 n+2)\left(s_{n+1}\right)^{-1} k x \quad(M \in D),
$$

where $\mu_{1}, \mu_{2} \in \mathscr{P}+$ and

$$
\mu_{2}=-(n+1) \sum_{j=1}^{\infty} \lim _{r \rightarrow 0+} \sup _{r}\left\{r^{n+1} \mathscr{M}\left(u, P_{j}, r\right)\right\} \delta_{j},
$$

$\delta_{j}$ being the unit Dirac measure concentrated at $P_{j}$.

We have stated the result for the case where the countable exceptional set is infinite. It is trivial to modify the statement and proof to cover the case where the countable exceptional set is finite or empty.

Since $u \in \mathscr{H}$, we can write $u(M)=I_{\mu}(M)+c x$ for each $M$ in $D$, where $\mu \in \mathscr{F}$ and $c$ is a real number. Suppose that $\varepsilon>0$ and for each positive integer $j$ put $\alpha_{j}=\mu^{-}\left(\left\{P_{j}\right\}\right)+\varepsilon 2^{-j}$. Let

in $D$. Since

$$
w=u+\sum_{j=1}^{\infty} \alpha_{j} I_{\delta_{j}}
$$

$$
\sum_{j=1}^{\infty} \alpha_{j} \int_{\partial D}(1+|P|)^{-n-1} d \delta_{j}(P) \leqq \int_{\partial D}(1+|P|)^{-n-1} d \mu^{-}(P)+\varepsilon \sum_{j=1}^{\infty} 2^{-j}<+\infty,
$$

$w \in \mathscr{H}$. Let $w(M)=I_{\eta}(M)+c^{\prime} x$ for each $M$ in $D$, where $\eta \in \mathscr{F}$ and $c^{\prime}$ is a real number.

Now let $E$ be the set of points of $\partial D$ at which the limit (13) fails to exist or is negative. By Lemma $2, \lambda(E)=0$. Since $w \geqq u$ in $D$, we have

$$
\liminf _{r \rightarrow 0+} r \mathscr{M}(w, P, r) \geqq 0 \quad(P \in \partial D \backslash E) .
$$

Since

$$
\eta\left(\left\{P_{j}\right\}\right)=\mu\left(\left\{P_{j}\right\}\right)+\alpha_{j}>0
$$


for each positive integer $j$, we have, by [1, Theorem 2, Corollary]

Hence

$$
\lim _{r \rightarrow 0+} r^{n+1} \mathscr{M}\left(I_{\eta}, P_{j}, r\right)>0 .
$$

$$
r \mathscr{M}\left(w, P_{j}, r\right) \rightarrow+\infty \quad(r \rightarrow 0+; j=1,2, \ldots) .
$$

By Lemma 3, there exists a positive harmonic function $v$ in $D$ such that $r \mathscr{M}(v, P, r) \rightarrow$ $+\infty$ as $r \rightarrow 0+$ for each $P$ in $E$. If $P \in E \backslash\left\{P_{1}, P_{2}, \ldots\right\}$, then

$$
\limsup _{r \rightarrow 0+} r \mathscr{M}(w, P, r) \geqq \limsup _{r \rightarrow 0+} r \mathscr{M}(u, P, r)>-\infty,
$$

so that

$$
\limsup _{r \rightarrow 0+} \frac{\mathscr{M}(w, P, r)}{\mathscr{M}(v, P, r)} \geqq 0 \quad\left(P \in E \backslash\left\{P_{1}, P_{2}, \ldots\right\}\right)
$$

By Lemmas 1 (with $v \equiv 0, d=1$ ) and 4,

$$
\mathscr{M}(w, \mathcal{O}, r) \rightarrow(2 n+2)^{-1} s_{n+1} c^{\prime} \quad(r \rightarrow \infty) .
$$

From (14), (15), (16), (17) and Theorem 6, we find that $w(M) \geqq c^{\prime} x$ for each $M$ in $D$. Hence, letting $\varepsilon \rightarrow 0+$, we obtain

Hence

$$
u(M)+\sum_{j=1}^{\infty} \mu^{-}\left(\left\{P_{j}\right\}\right) I_{\delta_{j}}(M)-c^{\prime} x \geqq 0 \quad(M \in D) .
$$

$$
u(M)=I_{\mu_{1}}(M)-\sum_{j=1}^{\infty} \mu^{-}\left(\left\{P_{j}\right\}\right) I_{\delta_{j}}(M)+c x \quad(M \in D)
$$

for some $\mu_{1} \in \mathscr{F}+$.

From [1; Theorem 2, Corollary] it follows that

$$
r^{n+1} \mathscr{M}\left(I_{\mu}, P_{j}, r\right) \rightarrow(n+1)^{-1} \mu\left(\left\{P_{j}\right\}\right) \quad(j=1,2, \ldots) .
$$

Hence, by Lemma 4,

$$
\mu\left(\left\{P_{j}\right\}\right)=(n+1) \lim _{r \rightarrow 0+} r^{n+1} \mathscr{M}\left(u, P_{j}, r\right) .
$$

Since $r \mathscr{M}\left(u, P_{j}, r\right) \rightarrow-\infty$ for each $j$, the limit in (19) is non-positive. Hence $\mu\left(\left\{P_{j}\right\}\right)$ in (19) can be replaced by $-\mu^{-}\left(\left\{P_{j}\right\}\right)$, and it follows from (18) that

$$
u(M)=I_{\mu_{1}}(M)+(n+1) \sum_{j=1}^{\infty} \lim _{r \rightarrow 0+}\left\{r^{n+1} \mathscr{M}\left(u, P_{j}, r\right)\right\} I_{\delta_{j}}(M)+c x \quad(M \in D) .
$$

Finally, by Lemmas 1 (with $v \equiv 0, d=1$ ) and 4

$$
\mathscr{M}(u, \mathcal{O}, r) \rightarrow(2 n+2)^{-1} s_{n+1} c,
$$

so that $c=(2 n+2)\left(s_{n+1}\right)^{-1} k$, as required. 


\section{References}

[1] Armitage, D. H.: Normal limits, half-spherical means and boundary measures of half-space Poisson integrals. - Hiroshima Math. J. 11, 1981, 235-246.

[2] Armitage, D. H.: A Nevanlinna theorem for superharmonic functions in half-spaces, with applications. - J. London Math. Soc. (2) 23, 1981, 137-157.

[3] Besicovitch, A. S.: A general form of the covering principle and relative differentiation of additive functions II. - Proc. Cambridge Philos. Soc. 41, 1945, 1-10.

[4] Bruckner, A. M., A. J. Lohwater, and F. Ryan: Some non-negativity theorems for harmonic functions. - Ann. Acad. Sci. Fenn. Ser. A I 452, 1969, $1-8$.

[5] Dinghas, A.: Über positive harmonische Funktionen in einem Halbraum. - Math. Z. 46, $1940,559-570$.

[6] FletT, T. M.: On the rate of growth of mean values of holomorphic and harmonic functions. - Proc. London Math. Soc. (3) 20, 1970, 749-768.

[7] Kuran, Ü.: Study of superharmonic functions in $R^{n} \times(0,+\infty)$ by a passage to $R^{n+3}$. - Proc. London Math. Soc. (3) 20, 1970, 276-302.

[8] Kuran, Ü.: A criterion of harmonic majorization in half-spaces. - Bull. London Math. Soc. 3, 1971, 21-22.

[9] Lohwater, A. J.: A uniqueness theorem for a class of harmonic functions. - Proc. Amer. Math. Soc. 3, 1952, 278-279.

[10] TsujI, M.: Potential theory in modern function theory. - Maruzen Co., Ltd., Tokyo, 1959.

[11] WAtson, N. A.: Initial and relative limiting behaviour of temperatures on a strip. - to appear.

The Queen's University of Belfast

Department of Pure Mathematics

Belfast BT7 1NN

Northern Ireland

Received 16 January 1981 